\title{
Cooling of Molecular Ion Beams
}

\author{
A. Wolf ${ }^{\text {a,* }}$ S. Krohn ${ }^{\text {a }}$ H. Kreckel ${ }^{\text {a }}$ L. Lammich ${ }^{\text {a }}$ M. Lange ${ }^{\text {a }}$ \\ D. Strasser ${ }^{b}$ M. Grieser ${ }^{a}$ D. Schwalm ${ }^{a}$ D. Zajfman ${ }^{b, a}$ \\ a Max-Planck-Institut für Kernphysik, Postfach 103980, D-69029 Heidelberg, \\ Germany \\ ${ }^{\mathrm{b}}$ Department of Particle Physics, Weizmann Institute of Science, Rehovot, Israel
}

\begin{abstract}
An overview of the use of stored ion beams and phase space cooling (electron cooling) is given for the field of molecular physics. Emphasis is given to interactions between molecular ions and electrons studied in the electron cooler: dissociative recombination and, for internally excited molecular ions, electron-induced ro-vibrational cooling. Diagnostic methods for the transverse ion beam properties and for the internal excitation of the molecular ions are discussed, and results for phase space cooling and internal (vibrational) cooling are presented for hydrogen molecular ions.
\end{abstract}

Key words:

PACS:

\section{Introduction}

Molecular ions and their interactions present a broad spectrum of systems and processes, which are of large interest to study for both basic and applied science. For adjacent scientific fields such as astrophysics [1], plasma physics [2], and even biophysics [3], benchmark experimental data on molecular ion processes help to develop reliable theoretical modeling methods. For example, the evolution of cold interstellar molecular clouds (which are important star forming regions) is driven by molecular ion chemistry [4], and the reactions of such ions with, e.g., electrons determine their abundances and internal excitations and finally the outcome of reaction chains, found to produce in space even complex organic molecular species. Regarding basic science, the detailed understanding of quantum mechanical many-body dynamics can be advanced

* Corresponding author; email: A.Wolf@mpi-hd.mpg.de 
by controlled, possibly state-resolved experiments on electron, photon and heavy-particle collisions involving atoms and molecules, in close interaction with the development of theoretical methods, which have to rely on suitable approximations to describe the quantum dynamics of three or more interacting particles. In fact, regarding the detailed understanding of molecular processes and interactions, even small systems such as the hydrogen molecular ion are still of great interest both experimentally and theoretically.

Molecular ion beams of $\mathrm{keV}$ and $\mathrm{MeV}$ energies have been used for spectroscopy and collision physics in a variety of studies, in particular for laser spectroscopy [5], single-pass electron-ion merged beams experiments [6], studies of heavy particle collisions and charge transfer [7], and Coulomb explosion experiments [8]. The majority of these studies does not use beam storage. However, when ion storage rings were put into service for atomic physics experiments, it soon became clear that they offer important advantages also for investigations of molecular systems. Meanwhile, the molecular ions for which beam storage has been realized span a very wide range of masses, from small systems such as $\mathrm{H}_{2}^{+}$ via polyatomic species such as, e.g., $\mathrm{H}_{3} \mathrm{O}^{+}$[9] to clusters $\left(\mathrm{C}_{60}^{-}\right)$[10] and large biomolecules [11]. Smaller systems as characterized by the first two examples were stored mostly as singly charged, positive ions in magnetic storage rings [12] at energies in the few-MeV range; some experiments were also performed with negative and with doubly charged positive molecular ions. The limits in magnetic rigidity imposed by using magnetic deflecting fields motivated the development of electrostatic storage rings [13], which at energies in the keV range have stored mostly heavy molecules, clusters and biomolecules; these rings recently have become particularly powerful tools for the study of heavy molecules by their combination with electrospray ion sources [14].

For the $\mathrm{MeV}$ molecular ion beams in magnetic storage rings, the only efficient phase space cooling method realized so far is electron cooling. Electron cooling of $\mathrm{H}_{2}^{+}$ions was demonstrated already in 1991 at the TARN II facility in Tokyo [15]; also the first recombination signal from $\mathrm{H}_{2}^{+}$ions in an electron cooler was reported in this work. Storage ring experiments dedicated to molecular recombination [16-18] followed soon after and started an intensive program of molecular recombination studies at heavy-ion storage-ring facilities. In electrostatic storage rings with typical ion energies in the $\mathrm{keV}$ region, most experiments so far were performed without applying phase space cooling. Within the field of electron cooling, its application to keV ion beams is characterized by very low electron acceleration voltages in the few eV range. Very recent studies on electron cooling at electrostatic storage rings were reported at this conference [19].

In addition to the translational degrees of freedom whose temperature is reduced by the conventional phase space cooling, also the internal (vibrational and rotational) degrees of freedom are relevant for a molecular ion beam. 
Molecular ions injected into a storage ring with initial vibrational excitation often relax to the vibrational ground state by emitting infrared radiation; typical time constants for such radiative relaxation in the vibrational degree of freedom are of the order of $0.1-1 \mathrm{~s}$. This produces a vibrationally cold stored ion beam, in contrast to the rather strong vibrational excitation found in molecular ion beams produced directly from most ion sources. Many experiments with molecular ions in magnetic storage rings profit from this internal cooling of the stored beam [20]. However, not all species efficiently cool by infrared emission; thus, among the various isotopomers of the hydrogen molecular ion, $\mathrm{HD}^{+}$could be well studied in its vibrational ground state [21] as it shows rapid radiative relaxation, while such relaxation is essentially absent for both $\mathrm{H}_{2}^{+}$and $\mathrm{D}_{2}^{+}$. For the heavier and more complex molecular ions in electrostatic storage rings, including clusters and biomolecules, the internal relaxation also played a central role in the experimental studies; thus, time constants for the radiative cooling of fullerene anions $\left(\mathrm{C}_{60}^{-}, \mathrm{C}_{56}^{-}\right)$were determined [10].

Apart from the natural cooling by spontaneous infrared emission, one has also started to apply external interactions for ro-vibrational cooling, such as collisions in the merged cold electron beam used for electron cooling. Great possibilities open up in connection with stored molecular ion beams as soon as sensitive diagnostic methods for the ro-vibrational populations in these beams become available. Electron-ion interactions in the electron cooler, considering recombination fragment detection and the transverse phase space cooling of molecular ion beams, will be discussed in the following, before turning to vibrational diagnostics on stored ion beams and studies yielding rates for collision induced cooling of internal molecular degrees of freedom.

\section{Electron interactions with molecular ions}

A class of experiments with molecular ions where the ion storage technique is most powerful are studies of their interations with electrons at well defined energies, performed in the electron cooling device. The main processes occurring in electron collisions of the simplest molecular ion, $\mathrm{H}_{2}^{+}$, are illustrated in Fig. 1. Dissociative recombination leads to two neutral $\mathrm{H}$ atoms and the electronic binding energy set free by the neutralization of the positive ion is given to these fragments as a kinetic energy release (KER) amounting, e.g., to $E_{k} \sim 1.5 \mathrm{eV}$ in the special case of Fig. 1 . This process is exothermic and has high cross sections at low electron energies; the dissociative recombination rate usually is more than three orders of magnitude higher than the radiative recombination rate of singly charged atomic ions at similar electron densities and relative energies. In fact, storage lifetimes of molecular ion beams, anyway shorter than for comparable atomic ion beams because of dissociation by rest gas collisions, can be reduced substantially by dissociative recombination 
(typical beam decay time constants are $\sim 5-10 \mathrm{~s}$ ). Dissociative recombination rates also depend significantly on the initial vibrational state of the ion.

A second exothermic process, electron-induced de-excitation, can occur in collisions with internally excited molecular ions, as illustrated in Fig. 1 for the case of vibrational excitation. It leads to a vibrational cooling of the molecular ions and in principle opens up the possibility to extend 'electron cooling' also to internal degrees of freedom of molecular ion beams. Theoretical calculations indicate that the rotational degrees of freedom are cooled by electron collisions as well, in fact at similar rates as the vibrations [22].

The collinear electron-ion interaction zone applied for electron cooling provides a very useful laboratory for studying the inelastic electron-ion collisions introduced here. In particular, with present electron coolers well controlled interaction energies can be realized down to a few milli-eV, corresponding to electron temperatures of only a few tens of Kelvin [23,24]. Hence, dissociative recombination can be studied at high energy resolution over a wide span of energies and also under conditions similar to those in, e.g., cold astrophysical environments. Detectors for the neutral fragments are installed as shown in Fig. 2. Energy sensitive detectors are used to measure recombination rates, identifying recombination events by the quasi-simultaneous arrival of all heavy constituents of the original molecule. Using ion and electron current measurements, the dissociative recombination rates can be normalized to yield rate coefficients, which can be compared to corresponding theoretical quantities obtained from the electron velocity distribution and calculated recombination cross sections. Detailed storage ring measurements have been performed on the DR rate coefficients of many species, including benchmark systems such as $\mathrm{HD}^{+}[21]$.

In addition to these rate measurements, imaging detectors are used to analyze the dissociation dynamics. Mostly, the transverse momenta of all recombination fragments are recorded event by event using a spatially resolving multi-hit detector (two-dimensional fragment imaging) [25]. Space and time-resolving multi-hit detectors also have been used in some cases for three-dimensional fragment imaging.

A typical pattern of neutral fragments from dissociative recombination of stored molecular ions in the electron cooler, as observed on an imaging detector, is illustrated in Fig. 3. The spatial distribution of the fragments is dominated by their transverse momenta acquired in the dissociation process. The largest transverse distances from the beam center reflect the energy release $E_{k}$. In fact, the distribution of transverse fragment distances in the case shown can be analyzed to derive the relative contributions to the recombination signal from $\mathrm{D}_{2}^{+}$ions in various vibrational levels $v$, as the final electronic excitation of the fragments corresponds to well known energy levels $(n=2$ in 
Fig. 1) and the electron energy is small ( $\sim 12 \mathrm{meV})$, making $E_{k}$ dependent on the initial $v$ level only.

For beams of atomic ions, notably protons, the spatial profile of the neutral atoms produced by recombination in the electron cooler offers a useful diagnostic for monitoring the transverse ion beam properties (mainly the angular divergence) during the phase space cooling [26,27]. In the case of molecular ions, the profiles of atomic recombination fragments cannot be used directly for this purpose because of the significant KER in the dissociation process. However, provided the atomic masses associated with each fragment hit on the imaging detector are known, the center-of-mass (c.m.) of all fragment hit positions for a given recombination event reflects the motion of the molecular ion before it captured an electron in the cooler; hence, the c.m. distributions for a molecular ion beam give the information equivalent to the neutral hydrogen diagnostic for a proton beam. The transverse electron cooling of a $\mathrm{D}_{2}^{+}$ ion beam at the storage ring TSR is illustrated in Fig. 4. The time dependence of the c.m. distribution demonstrates efficient electron cooling down to a divergence (FWHM) of $<0.12 \mathrm{mrad}$ (spot of $0.6 \mathrm{~mm}$ at $\sim 5.5 \mathrm{~m}$ distance from the electron cooler). Reversely, efficient transverse phase space cooling of molecular ion beams is very helpful in the analysis of fragment imaging data, as all events with correct kinematical identification are expected to lie in a narrow range of the c.m. distribution defined by the primary molecular ion beam, while incorrectly analyzed events (such as random coincidences from independent ionic dissociation events yielding only one neutral fragment each) will lie outside this range and can be reliably suppressed by a cut on the c.m. coordinate.

\section{Cooling of internal degrees of freedom}

Apart from recombination, electron-induced de-excitation of internal molecular motion (see Fig. 1) is the second exothermic, inelastic collision process that accompanies the electron cooling of molecular ions. While recombination can be observed and studied in a rather straightforward way by detecting the neutral products, measurements on electron-induced ro-vibrational de-excitation require suitable diagnostic methods to be applied on the stored molecular ions. Electron-induced cooling only leads to significant effects if the internal molecular motion does not relax radiatively. This holds for the symmetric hydrogen species $\mathrm{H}_{2}^{+}$and $\mathrm{D}_{2}^{+}$, where the center of charge is pinned to the center of mass during vibrations and rotations. For the asymmetric species $\mathrm{HD}^{+}$this is not the case and hence its vibrations, as well as its high rotations, cool rapidly by spontaneous decay. Apart from a few initial studies on rotational cooling, mentioned in the Outlook, only vibrational cooling has been studied so far, using essentially three different methods. 
The first method makes use of the energy dependence of the dissociative recombination cross section at elevated electron energies. In the range of $\sim 5-15$ $\mathrm{eV}$, the shape of the cross section [16-18] for many ions reflects the distribution of internuclear distances according to the Franck-Condon principle, governing electronic transitions between molecular potential curves. Cross sections as a function of the storage time were observed for $\mathrm{D}_{2}^{+}$and $\mathrm{H}_{2}^{+}$ions already in early work at storage rings, including CRYRING [28,29] where significant increases of the relative population in $v=0$ were found during storage times of up to $\sim 40 \mathrm{~s}$. It was first suspected that the effect would be due to the preferential destruction of vibrationally excited ions either by collisions with rest gas molecules [28] or by the dissociative recombination process itself [29]. Later, similar measurements at TARN II [30] finally demonstrated that indeed $\mathrm{H}_{2}^{+}$ions in higher vibrational states were converted into ground-state $(v=0)$ ions during electron cooling, giving evidence for electron-induced vibrational de-excitation ("super-elastic collisions").

As a second diagnostic method for the vibrational excitation of the stored ions, recombination fragment imaging can be used (see Fig. 3). As discussed in Section 2, the fragment distance distributions reflect the relative contributions from ions in various vibrational levels to the recombination signal. These relative recombination rates were investigated at the TSR during the radiative cooling of $\mathrm{HD}^{+}$ions [31] and later for $\mathrm{H}_{2}^{+}$and $\mathrm{D}_{2}^{+}$in connection with the Coulomb explosion imaging studies described below. It should be pointed out that both the first and the second method do not directly yield the relative populations of vibrational levels. These quantities can only be obtained if the recombination rate coefficients are known for each initial $v$ state. Hence, taken by themselves, both methods need theoretical calculations in order to extract relative populations of vibrational levels. Yet, recent experiments at TARN II [32] used recombination fragment imaging alone to study the vibrational relaxation of $\mathrm{H}_{2}^{+}$and $\mathrm{D}_{2}^{+}$ions, deriving with the help of theoretical rate coefficients a population fraction of $>90 \%$ for the $v=0$ state after $26 \mathrm{~s}$ of electron cooling.

As a third diagnostic method, foil-induced Coulomb explosion imaging of fast molecular ions [8] can be used to directly determine population fractions for vibrational levels, at least for light species such as di- and triatomic hydrogen and, e.g., $\mathrm{He}_{2}^{+}$. The principle of the method is shown in Fig. 5. Coulomb explosion imaging of molecular ions slowly extracted from an ion storage ring can be used to follow the time dependence of vibrational populations in a stored ion beam. This approach was realized at the TSR [33] and used to study the vibrational excitation and the structure of several light molecular ions. Fragment energy distributions obtained by Coulomb explosion imaging of $\mathrm{HD}^{+}$extracted from the TSR for different time windows after injection are shown in Fig. 6. It is seen that the shape and the width of the fragment energy distribution change strongly during the first $400 \mathrm{~ms}$ of ion storage, as excited 
vibrational levels decay radiatively finally leaving only $\mathrm{HD}^{+}(v=0)$ ions in the stored beam. Simulated fragment energy distributions, calculated for specific $v$ states and summed up with variable weight factors, are fitted to the measured distibutions; the weight factors resulting from the fit directly yield the vibrational populations, some of which are given in the Figure. Theoretical predictions of radiative vibrational cooling rates are in good agreement with the time dependent populations which could be determined for the states $v=0-11$ of $\mathrm{HD}^{+}[34]$.

To demonstrate collision-induced vibrational cooling, the time dependent vibrational populations measured by Coulomb explosion imaging as measured during electron cooling of $\mathrm{D}_{2}^{+}[35]$ are shown in Fig. 7. It should be emphasized that these cooling curves, beside electron-induced vibrational cooling, also reflect the vibrational state dependence of any loss processes experienced by the molecular ions. A significant effect due to rest gas collisions can be excluded, as constant vibrational populations are observed in the absence of the electron beam [36]. However, also dissociative recombination contributes to ion loss, and changes of the vibrational populations due to selective depletion of different $v$ levels must be estimated. Similar to earlier studies on $\mathrm{HD}^{+}$ [31], Coulomb explosion imaging was combined with recombination fragment imaging, as described above, which allowed vibrational-state specific recombination rate coefficients to be determined simultaneously. Absolute recombination rate coefficients were hence obtained for several vibrational levels $\left(v=0-7\right.$ for $\left.\mathrm{D}_{2}^{+}\right)$. These quantities were then applied in a rate equation model together with electron-induced vibrational cooling rates (with a restriction to transitions $v \rightarrow v-1$ ) to fit the time dependent vibrational populations of Fig. 7. The fit finally yielded [35] vibrational cooling rate coefficients in the range from $(36 \pm 7) \times 10^{-8} \mathrm{~cm}^{3} \mathrm{~s}^{-1}$ for $v=1 \rightarrow 0$ up to $(220 \pm 44) \times 10^{-8} \mathrm{~cm}^{3} \mathrm{~s}^{-1}$ for $v=5 \rightarrow 4$. In comparison, the $\mathrm{D}_{2}^{+}$recombination rate coefficients for $v \leq 6$ are $<10 \times 10^{-8} \mathrm{~cm}^{3} \mathrm{~s}^{-1}$. Electron-induced transitions where $v$ changes by $|\Delta v|>1$, theoretically predicted to be much smaller than those with $|\Delta v|=1$, were not included in the fit. Athough the experimental results have triggered new calculations, theory so far cannot reproduce the large experimental vibrational cooling rates, remaining typically a factor of 5-8 below the observed values, while the dissociative recombination rate coefficients are rather well reproduced.

\section{Outlook}

The experiments described above show that, even in the absence of radiative interactions, the internal excitations of stored molecular ions evolve significantly on time scales of $\sim 10-30 \mathrm{~s}$. The principal limit for internal cooling appears to be given be the temperature of the relevant "heat bath", presently 
down to $\sim 10 \mathrm{~K}$ for the electron cooler and $\sim 300 \mathrm{~K}$ if radiative interactions dominate (blackbody field). However, the population of excited levels corresponding to these temperature limits only becomes relevant if not only vibrations, but also rotations are considered. Diagnostic measurements on the rotational excitation in stored ion beams are still scarce. Using laser spectroscopy, rotational cooling of $\mathrm{CH}^{+}$ions down to the $300 \mathrm{~K}$ radiation temperature of the storage ring enclosure, without significant heating through rest gas collisions or other external influences, could be demonstrated [37]. Recombination fragment imaging also yields information on rotational excitation of the stored ions [38], but is not sensitive enough to analyze temperatures below $\sim 500 \mathrm{~K}$. Recently, variations of the rate coefficient for dissociative recombination were observed for long storage times of triatomic hydrogen ions and interpreted as rotational cooling of the stored ion beam [39]. In principle, the low temperatures available in electron coolers and the relatively weak coupling of low rotations to the radiation field could make it possible to reach equilibrium temperatures below the $300 \mathrm{~K}$ radiative background in a storage ring by the continuous effect of electron collisions. Using this approach, as well as cryogenic electrostatic storage rings providing radiative cooling [40], intense development is under way to produce internally cold molecular ion beams for well controlled experiments.

\section{Acknowledgments}

This work was partly funded by the German Federal Minister for Education, Science, Research and Technology (BMBF) in the framework of the GermanIsraeli Project Cooperation on Future-Oriented Topics (DIP), by the German Israel Foundation for Scientific Research (GIF) under contract I-70755.7/2001 and by the European Community within the Research Training Network "Electron Transfer Reactions".

\section{References}

[1] K. Kirby, Phys. Scr. T59 (1995) 59-64.

[2] R. L. Janev, in: M. Larsson, J. B. A. Mitchell, I. F. Schneider (Eds.), Dissociative Recombination: Theory, Experiment and Applications IV, World Scientific, Singapore, 2000, pp. 40-47.

[3] R. A. Zubarev, in: M. Larsson, J. B. A. Mitchell, I. F. Schneider (Eds.), Dissociative Recombination: Theory, Experiment and Applications IV, World Scientific, Singapore, 2000, pp. 214-217.

[4] A. Sternberg, A. Dalgarno, Astrophys. J. 99 (1995) 565. 
[5] S. G. Cox, A. D. J. Critchley, I. R. McNab, F. E. Smith, Meas. Sci. Technol. 10 (1999) R101-R128.

[6] D. Auerbach, R. Cacak, R. Caudano, C. J. Keyser, T. D. Gaily, J. B. A. Mitchell, J. W. McGowan, S. F. J. Wilk, J. Phys. B 10 (1977) 3797.

[7] A. B. V. der Kamp, J. H. M. Beijersbergen, P. C. Cosby, W. J. V. der Zande, J. Phys. B 27 (20) (1994) 5037-5053.

[8] Z. Vager, R. Naaman, E. P. Kanter, Science 244 (1989) 426.

[9] A. B. V. der Kamp, J. H. M. Beijersbergen, P. C. Cosby, W. J. V. der Zande, Astrophys. J. 483 (1997) 531-540.

[10] J. U. Andersen, C. Gottrup, K. Hansen, P. Hvelplund, M. O. Larsson, Eur. Phys. J. D 17 (2001) 189-204.

[11] S. Boyé, H. Krogh, I. B. Nielsen, S. B. Nielsen, S. U. Pedersen, U. V. Pedersen, L. H. Andersen, A. F. Bell, X. He, P. J. Tong, Phys. Rev. Lett. 90 (2003) 118103.

[12] A. Wolf, in: H. F. Beyer, V. P. Shevelko (Eds.), Atomic Physics With Heavy Ions, Springer, Berlin, 1999, pp. 1-29.

[13] S. P. Møller, Nucl. Instrum. Methods A 394 (1997) 281.

[14] J. U. Andersen, P. Hvelplund, S. B. Nielsen, S. Tomita, H. Wahlgreen, S. P. Møller, U. V. Pedersen, J. S. Forster, T. J. D. Jørgensen, Rev. Sci. Instrum. 73 (2002) 1284-1287.

[15] T. Tanabe, K. Noda, T. Honma, M. Kodaira, K. Chida, T. Watanabe, A. Noda, S. Watanabe, A. Mizubuchi, M. Yoshizawa, T. Katayama, H. Muto, Nucl. Instrum. Methods Phys. Res. A 307 (1991) 7.

[16] T. Tanabe, I. Katayama, N. Inoue, K. Chida, Y. Arakaki, T. Watanabe, M. Yoshizawa, S. Ohtani, K. Noda, Phys. Rev. Lett. 70 (1993) 422.

[17] P. Forck, M. Grieser, D. Habs, A. Lampert, R. Repnow, D. Schwalm, A. Wolf, D. Zajfman, Phys. Rev. Lett. 70 (1993) 426.

[18] M. Larsson, H. Danared, J. R. Mowat, P. Sigray, G. Sundström, L. Broström, A. Filevich, A. Källberg, S. Mannervik, K. G. Rensfelt, S. Datz, Phys. Rev. Lett. 70 (1993) 430.

[19] T. Tanabe, report at this conference.

[20] M. Larsson, Annu. Rev. Phys. Chem. 48 (1997) 151-179.

[21] A. Al-Khalili, et al., Phys. Rev. A (2003) accepted.

[22] A. Faure, J. Tennyson, Mon. Not. Roy. Astron. Soc. 325 (2001) 443-448.

[23] H. Danared, A. Källberg, G. Andler, L. Bagge, F. Österdahl, A. Paál, K.-G. Rensfelt, A. Simonsson, O. Skeppstedt, M. af Ugglas, Nucl. Instrum. Methods A 441 (2000) 123-133. 
[24] G. Gwinner, A. Hoffknecht, T. Bartsch, M. Beutelspacher, N. Eklöw, P. Glans, M. Grieser, S. Krohn, E. Lindroth, A. Müller, A. A. Saghiri, S. Schippers, U. Schramm, D. Schwalm, M. Tokman, G. Wissler, A. Wolf, Phys. Rev. Lett. $84(2000) 4822$.

[25] Z. Amitay, D. Zajfman, P. Forck, U. Hechtfischer, B. Seidel, M. Grieser, D. Habs, D. Schwalm, A. Wolf, Phys. Rev. A 54 (1996) 4032.

[26] G. I. Budker, N. S. Dikansky, V. I. Kudelainen, I. N. Meshkov, V. V. Parkhomchuk, D. V. Pestrikov, A. N. Skrisky, B. N. Sukhina, Part. Accel. 7 (1976) 197-211.

[27] M. Bell, J. Chaney, H. Herr, F. Krienen, P. Møller-Petersen, G. Petrucci, Nucl. Instrum. Methods 190 (1981) 237-255.

[28] M. Larsson, M. Carlson, H. Danared, L. Brostrom, S. Mannervik, G. Sundstrom, J. Phys. B 27 (1994) 1397-1406.

[29] W. J. van der Zande, J. Semaniak, V. Zengin, G. Sundström, S. Rosén, C. Strömholm, S. Datz, H. Danared, M. Larsson, Phys. Rev. A 54 (1996) 5010.

[30] T. Tanabe, H. Takagi, I. Katayama, K. Chida, T. Watanabe, Y. Arakaki, Y. Haruyama, M. Saito, I. Nomura, T. Honma, K. Noda, K. Hosono, Phys. Rev. Lett. 83 (1999) 2163-2166.

[31] Z. Amitay, A. Baer, M. Dahan, L. Knoll, M. Lange, J. Levin, I. F. Schneider, D. Schwalm, A. Suzor-Weiner, Z. Vager, R. Wester, A. Wolf, D. Zajfman, Science 75 (1998) 281.

[32] M. Saito, Y. Haruyama, T. Tanabe, I. Katayama, K. Chida, T. Watanabe, Y. Arakaki, I. Nomura, T. Honma, K. Noda, K. Hosono, Phys. Rev. A 61 (2000) 062707.

[33] R. Wester, F. Albrecht, M. Grieser, L. Knoll, R. Repnow, D. Schwalm, A. Wolf, A. Baer, J. Levin, Z. Vager, D. Zajfman, Nucl. Instrum. Methods A 413 (1998) 379-396.

[34] Z. Amitay, A. Baer, M. Dahan, J. Levin, Z. Vager, D. Zajfman, L. Knoll, M. Lange, D. Schwalm, R. Wester, A. Wolf, I. F. Schneider, A. Suzor-Weiner, Phys. Rev. A 60 (1999) 3769.

[35] S. Krohn, H. Kreckel, L. Lammich, M. Lange, J. Levin, D. Schwalm, D. Strasser, R. Wester, A. Wolf, D. Zajfman, in: Proceedings of the Symposium on Dissociative Recombination of Molecular Ions held by the Chemical Society of America, Chicago, Aug. 27-30, 2001, in print; S. Krohn et al., to be published.

[36] S. Krohn, Z. Amitay, A. Baer, D. Zajfman, M. Lange, L. Knoll, J. Levin, D. Schwalm, R. Wester, A. Wolf, Phys. Rev. A 62 (2000) 032713.

[37] U. Hechtfischer, Z. Amitay, P. Forck, J. L. M. Lange, M. Schmitt, U. Schramm, D. Schwalm, R. Wester, D. Zajfman, A. Wolf, Phys. Rev. Lett. 80 (1998) 28092812 . 
[38] D. Strasser, L. Lammich, H. Kreckel, S. Krohn, M. Lange, A. Naaman, D. Schwalm, A. Wolf, D. Zajfman, Phys. Rev. A 66 (2002) 032719.

[39] L. Lammich, D. Strasser, H. Kreckel, M. Lange, H. B. Pedersen, S. Altevogt, V. Andrianarijaona, H. Buhr, O. Heber, P. Witte, D. Schwalm, A. Wolf, D. Zajfman, submitted.

[40] E. g., T. Azuma, report at this conference. 


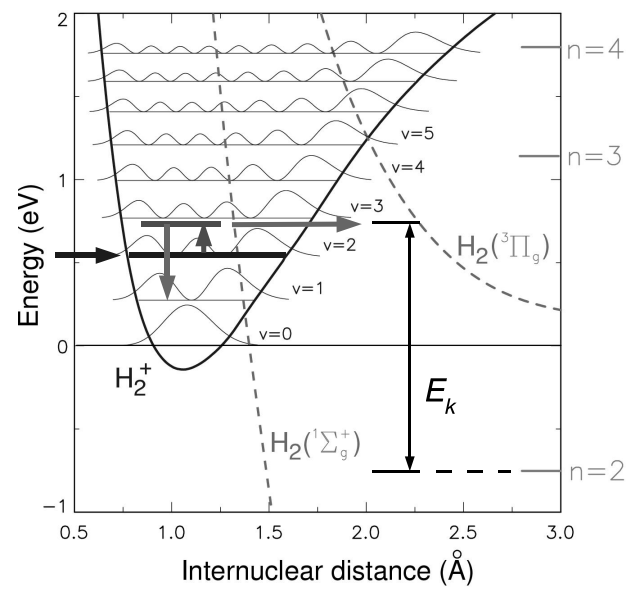

Fig. 1. Potential curves and vibrational energy levels of the $\mathrm{H}_{2}^{+}$molecular ion together with dissociating potentials of the neutral system $\mathrm{H}_{2}$. Relevant energy levels of the separated $\mathrm{H}$ atoms (with one atom in the $1 s$ ground state and the other in an excited state $n)$ are also indicated. Processes are shown for $\mathrm{H}_{2}^{+}(v=2)$ ions colliding with an electron of the energy marked by the upwards vertical arrow. Dissociative recombination along the $\mathrm{H}_{2}\left({ }^{1} \Sigma_{g}^{+}\right)$neutral potential yields two $\mathrm{H}$ atoms with a KER of $E_{k}$, while collision-induced vibrational de-excitation leaves an ion $\mathrm{H}_{2}^{+}(v=1)$ and an electron of the energy indicated by the downwards vertical arrow.

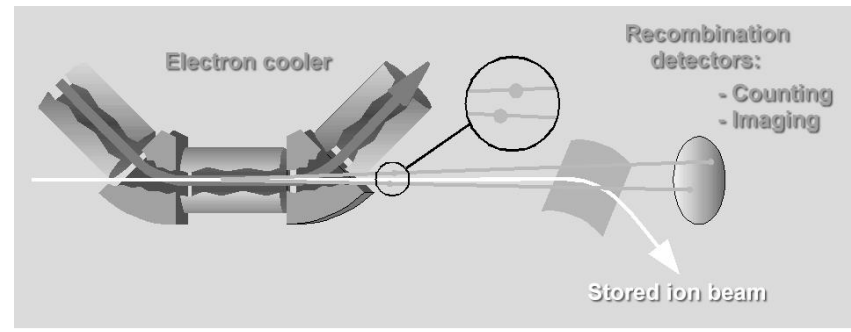

Fig. 2. Principle of recombination fragment counting and imaging in experiments with molecular ions at an electron cooler. 


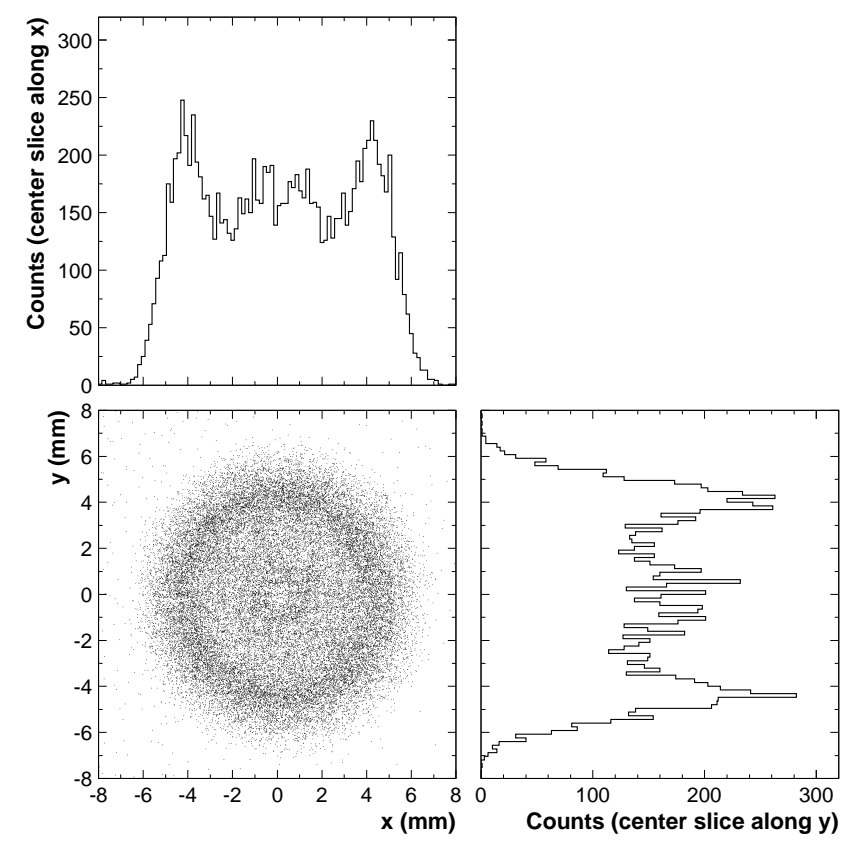

Fig. 3. Histograms of fragment hit positions for dissociative recombination during the electron cooling of a $1.9 \mathrm{MeV} \mathrm{D}_{2}^{+}$ion beam at the TSR facility in Heidelberg (storage time $>5 \mathrm{~s}$ ). Only events with two time-correlated neutral fragments are shown. The one-dimensional histograms show slices ( $\sim 3 \mathrm{~mm}$ wide) in the center of the hit distribution.

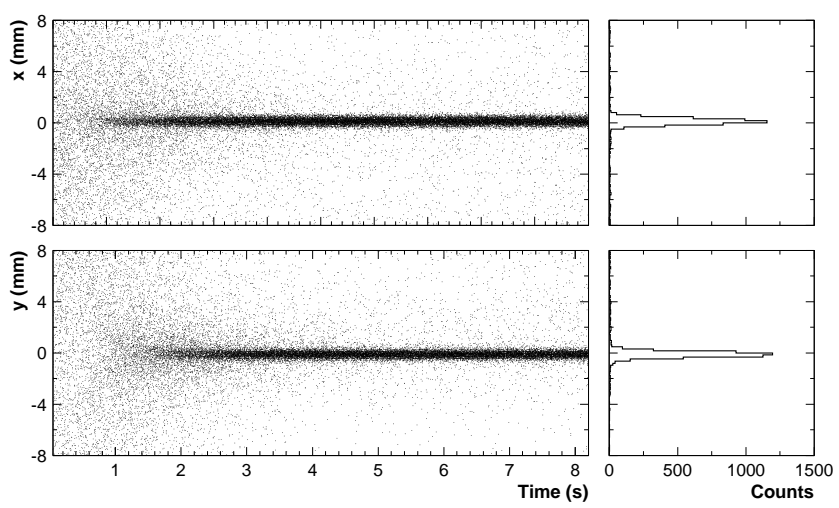

Fig. 4. Positions of the center of mass derived for correlated two-hit events similar to Fig. 3. A $1.9 \mathrm{MeV} \mathrm{D} \mathrm{D}_{2}^{+}$ion beam is cooled with an electron density of $5.6 \times 10^{6}$ $\mathrm{cm}^{-3}$ (transverse electron temperature $12 \mathrm{meV}$ ). The one-dimensional histograms $(0.16 \mathrm{~mm} / \mathrm{bin})$ show the distributions for the last slice $(\sim 0.5 \mathrm{~s}$ wide $)$ of the time scale (electron cooling equilibrium). 


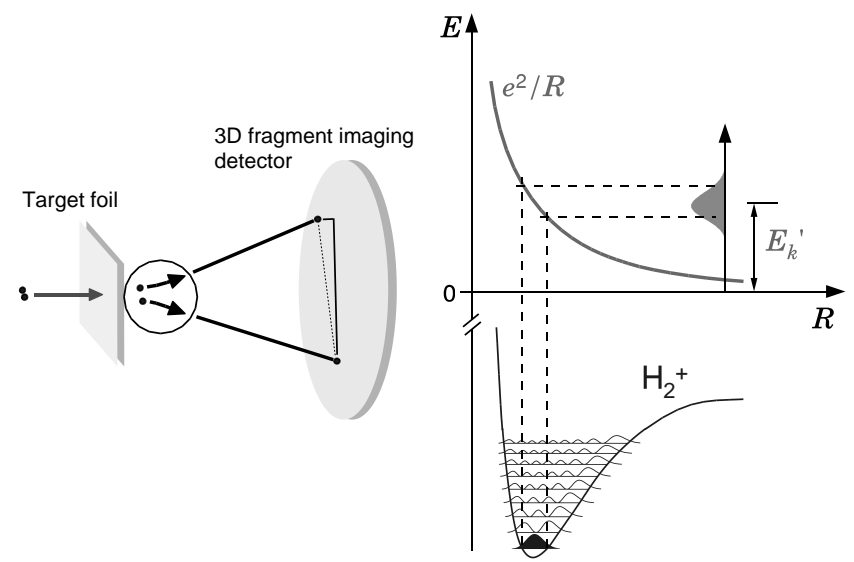

Fig. 5. Foil-induced Coulomb explosion imaging of molecular ions $[8,33]$ at velocities of $\sim 4$ a.u. $(E \sim 0.5 \mathrm{MeV} / \mathrm{u})$. Left: schematical arrangement with a $\sim 5 \mathrm{~nm}$ target foil (stripping time $\sim 0.1 \mathrm{fs}$; passage time $\sim 1 \mathrm{fs}$ ) and a time- and position-sensitive fragment imaging detector placed at $\sim 3 \mathrm{~m}$ distance (fragment distances of order $3 \mathrm{~cm}$ ). Right: "Projection" of the instantaneous nuclear coordinate in a diatomic molecule $\left(\mathrm{H}_{2}^{+}\right)$onto the Coulomb repulsion curve in the fast stripping process on entering the target foil; for an ensemble of events from molecular ions in a given $v$ state, the vibrational wave function (quantum mechanical probability density) is sampled through measurements of the Coulomb explosion energy release $E_{k}^{\prime}$.

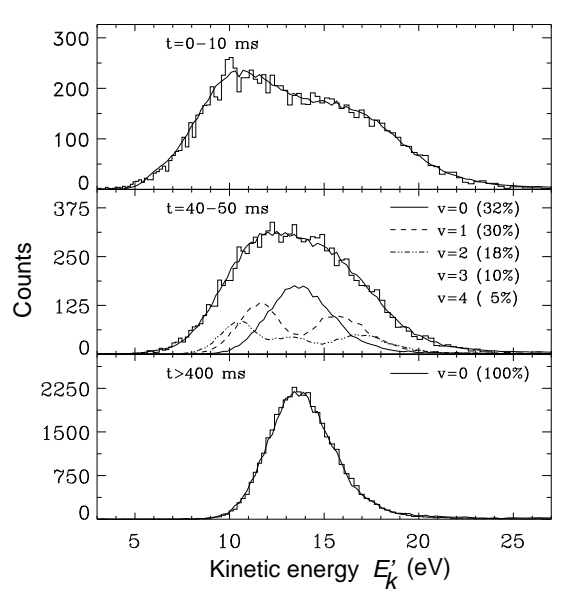

Fig. 6. Fragment energy distributions [31] for the foil-induced Coulomb explosion of $2.0 \mathrm{MeV} \mathrm{HD}{ }^{+}$ions at the TSR for different storage time windows as indicated. The smooth lines show simulated distributions fitted to the data (full lines) and components for individual vibrational levels (broken lines), for which relative populations in the stored $\mathrm{HD}^{+}$beam (as derived from the fit) are given. 


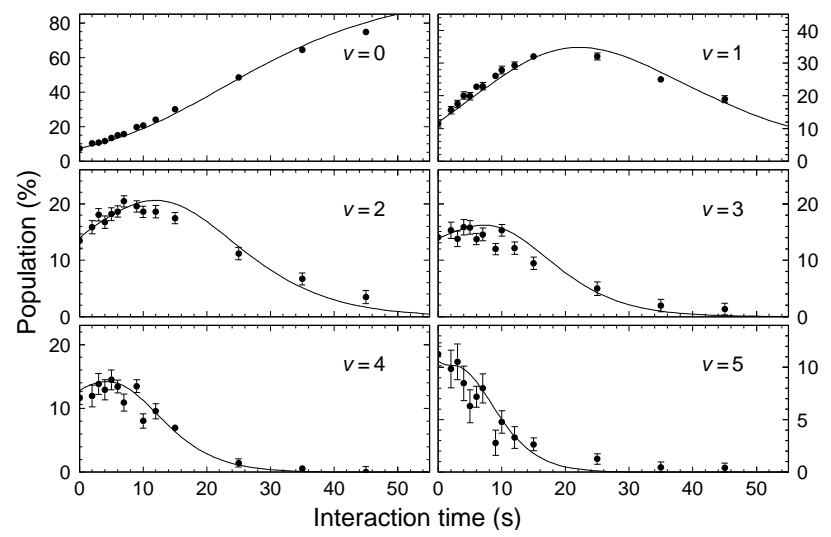

Fig. 7. Vibrational cooling of $1.9 \mathrm{MeV} \mathrm{D}_{2}^{+}$ions at the TSR during interaction with an electron beam (electron density $5.6 \times 10^{6} \mathrm{~cm}^{-3}$, transverse electron temperature $12 \mathrm{meV}$ ). The time dependent level populations are measured by Coulomb explosion imaging. 